\title{
Diabetes and brain damage: more (or less) than meets the eye?
}

\author{
Christopher M. Ryan
}

Published online: 18 August 2006

(C) Springer-Verlag 2006

Brain damage is now a well-established complication of diabetes, but its pathophysiological basis remains controversial. Until very recently, most research on this topic was devoted to demonstrating that hypoglycaemic events are the primary cause of neurocognitive dysfunction. This was a plausible hypothesis because the central nervous system (CNS) has very limited stores of glucose and other substrates, but neurons have a very high rate of glucose utilisation; any reduction in glucose availability would probably induce neuroglycopenia, ultimately leading to significant neuronal damage. Support for this view came from research demonstrating that profound hypoglycaemia could produce neuronal necrosis [1] and induce distinctive patterns of neuropathological [2] and neurocognitive [3] impairment in humans who had experienced very low blood glucose levels over an extended period of time. Fortunately, most diabetic patients never experience profound hypoglycaemia. Although some writers have speculated that recurrent episodes of moderately severe hypoglycaemia could induce a less severe degree of neurocognitive dysfunction $[4,5]$, most recent studies of children [6] and adults [7, 8] have failed to find compelling evidence for that possibility. Taken together, these findings suggest that there is not a linear relationship between falling blood glucose levels and permanent brain dysfunction. Rather, necrotising neuronal damage occurs only after the threshold for cerebral energy failure is reached-most typically when blood glucose values

\footnotetext{
C. M. Ryan $(\square)$

University of Pittsburgh School of Medicine,

3811 O'Hara Street,

Pittsburgh, PA 15213, USA

e-mail: ryancm@upmc.edu
}

fall below $1.5 \mathrm{mmol}$, and the electroencephalogram has reached an isoelectric (flat) state [9].

\section{Retinopathy predicts neurocognitive dysfunction}

If moderately low blood glucose levels are not sufficient to induce detectable brain damage, and if profound hypoglycaemia is a rare event, to what can we attribute the modest, but consistently reported, brain dysfunction found in many diabetic patients? A growing literature now suggests that functional and structural CNS changes may have a vascular basis, and reflect the occurrence of cerebral microangiopathy that is secondary to chronic hyperglycaemia and indicated by the presence of retinopathy. For example, when adults with type 1 diabetes were followed for 7 years, a significant decline in mental efficiency was found over time, but this was limited to those who either had clinically significant diabetic proliferative retinopathy when they entered the study or who subsequently developed diabetic proliferative retinopathy during the follow-up period; those without retinopathy showed no cognitive changes. The magnitude of cognitive decline was further, and independently, influenced by elevated systolic blood pressure and by duration of diabetes [10]. The presence of retinopathy-even relatively early retinopathy - has also been associated with cerebral white matter lesions [11] in otherwise healthy diabetic adults, and a recent exploratory analysis has suggested that increasing severity of retinopathy is related to reductions in cortical grey matter density [8].

Of perhaps even greater significance is the discovery that these effects are not limited to diabetic patients. Data from the Atherosclerosis Risk in Communities Study have now 
demonstrated that retinal microvascular abnormalities (particularly microaneurysms) are associated with exactly the same pattern of neurocognitive dysfunction - namely, psychomotor slowing - in middle-aged adults without diabetes [12]. Retinal abnormalities are also predictive of MRIdefined evidence of cerebral atrophy [13] and subclinical cerebral infarcts [14], and are especially pronounced in non-diabetic individuals with elevated blood pressure. Those associations, given the close anatomical and physiological relationships between the retinal and cerebral vascular networks [15], suggest that digitised fundus photography of retinal vessel diameters can provide a non-invasive assessment of the integrity of the cerebral microcirculation $[16,17]$.

\section{Cortical atrophy, cerebral hypoperfusion and diabetic proliferative retinopathy}

If chronic hyperglycaemia induces cerebral microangiopathy, and if the resulting vascular abnormalities lead to cerebral hypoperfusion, then diabetic patients with clinically significant retinopathy should show a greater degree of structural brain damage than those without retinopathy. In an elegant test of this hypothesis, Wessels and her colleagues employed a case-control design and found exactly that. As described in the present issue of Diabetologia [18], normotensive adults with advanced diabetic proliferative retinopathy had reduced grey matter density compared with a demographically similar group of childhood-onset diabetic adults with no clinically significant microvascular complications. Most noteworthy is the regional distribution of the grey matter density reductions. Rather than being spread diffusely across the cortex, these changes were limited to only a few areas, which tended to be in the more extreme, or 'watershed', areas of the distribution of the medial and posterior cerebral arteries. This is just what one would have predicted if cerebral hypoperfusion was a major factor contributing to the reduction in grey matter density seen in the diabetic patients with retinopathy, since these watershed areas are a prime site for cortical microinfarcts in other patient populations experiencing cerebrovascular insufficiency [19].

Structural changes of this sort ought to affect brain function as well, and in a separate report that included many of the same subjects, Wessels' group was able to demonstrate the existence of differences in brain activation during a cognitively demanding working memory task [20]. Normally, a deactivation of certain brain regions occurs during working memory, and this can be measured using functional MRI techniques. When brain activity was assessed twice-once during euglycae- mia and once during a hypoglycaemic clamp - those with diabetic proliferative retinopathy showed significantly less activation (indicative of subtle brain pathology) than those without retinopathy. Nevertheless, despite functional MRI evidence of changes in brain function during hypoglycaemia, cognitive function remained unaffected: those with diabetic proliferative retinopathy performed the working memory task as well as those without microangiopathy. It remains to be determined whether this preservation of cognitive performance is secondary to possible compensatory changes in cerebral blood flow [21] or in other neural or cerebrovascular processes that might develop over time in response to slowly evolving microvascular damage. Alternatively, it could merely reflect an insensitivity of the working memory task to very subtle alterations in CNS function.

\section{Is diabetes-related brain dysfunction a 'vasocognopathy'?}

For many reasons, it should not be particularly surprising to find a vascular basis for diabetes-related brain dysfunction. Blood pressure elevations, particularly increases in systolic blood pressure, are frequently found in patients with type 1 or type 2 diabetes. Such elevations are strongly associated with neurocognitive impairments [10] and cortical atrophy [22], and appear to have a synergistic effect insofar as individuals with both diabetes and elevated blood pressure tend to have the poorest neurocognitive outcomes [22-24]. Similarly, cerebral blood flow, especially in the frontal and frontotemporal brain regions, is reduced in diabetic patients $[25,26]$ as is cerebrovascular reactivity, with this latter effect being most pronounced in those with retinopathy [27] or hypertension [28]. Cerebral metabolism, as measured by proton magnetic resonance spectroscopy, has also been found to be significantly affected in diabetic patients and again, this is linked to the presence of hypertension [28] and retinopathy [29], with abnormalities being greatest among those with the poorest long-term metabolic control.

The development of degenerative dementias, such as Alzheimer's disease, has also been attributed to chronic brain hypoperfusion [30]. In a series of compelling theoretical papers, de La Torre [31, 32] has suggested that Alzheimer's disease may best be conceptualised as a 'vasocognopathy' ('vaso': vessel/blood flow; 'cogno': relating to cognition) that results from a cascade of haemodynamic abnormalities, including reduced cerebral flood flow, that lead to a decline in cerebral perfusion. According to that model, the resultant reduction in the delivery of glucose and oxygen initiates a neuroglial 
'energy crisis' that eventuates in mild cognitive impairment, more serious neurodegeneration and ultimately, in clinically significant dementia.

Many of the risk factors for Alzheimer's disease identified by de la Torre (e.g., hypertension, atherosclerosis, elevated cholesterol, ischaemic stroke, haemodynamic abnormalities, depression) are also co-morbid conditions associated with diabetes of long duration. Reasoning by analogy, one would predict that if diabetes-related cognitive impairment is primarily vascular in origin, then individuals with a childhood onset of diabetes ought to manifest a marked neurocognitive deterioration over a time period similar to that seen in adults who go on to develop Alzheimer's disease. Yet cross-sectional studies comparing cognitive functioning in diabetic and non-diabetic individuals [7] and longitudinal studies measuring cognitive decline over time in adults with type 1 diabetes [10] have typically found quite modest deficits that have never met criteria for 'clinically significance'. Although a number of studies have reported a somewhat greater risk of dementia among diabetic patients, these risks are also relatively small, and in the order of a 1.5-fold increased risk [33, 34].

Indeed, during the past 40 years there has been only one compelling report of profound neuropathological and neurological damage in type 1 diabetic patients, and that focused on 16 poorly controlled, middle-aged adults, who came to autopsy after 17-36 years of diabetes [35]. These patients showed evidence of cerebral angiopathy with enormously thickened walls in the arterioles and in the basement membrane of capillaries, accompanied by gross, diffusely distributed reductions in cortical grey matter, as well as white matter changes characterised by degeneration of myelin sheaths and axon cylinders. All were blind, or nearly blind, and all had neurological abnormalities that ranged from reflex abnormalities to profound dementia. Reske-Nielson and her associates aptly described their findings as evidence of 'diabetic encephalopathy', but while their work is consistent with the vasocognopathy model, there have been no other reports of such profound brain damage associated with type 1 diabetes in young and middle-aged adults. To some extent this may be a consequence of the dramatic improvements made in treating diabetes and reducing its micro- and macrovascular complications, but it may also reflect a failure, on the part of modern researchers, to enrol their most poorly controlled patients into research studies.

\section{Absence of progressive neurocognitive deterioration in older diabetic adults}

Because cerebral hypoperfusion and other vascular changes become more prominent during the course of normal ageing
[36, 37], one might also expect to find evidence of clinically significant impairment in older adults who have had type 1 diabetes for many years. Somewhat surprisingly, however, a recent report provides very little support for that possibility. Despite an average disease duration of 34 years and a high prevalence of microvascular complications, this group of older (52-77 years) diabetic individuals performed significantly more poorly than their demographically similar non-diabetic peers only on a single cognitive measure (speed of information processing), and the magnitude of that effect was small and not clinically significant [38]. Further, there were no between-group differences in MRI measures of cortical atrophy, white matter abnormalities or cerebral infarcts. This study suggests that diabetic patients have a remarkable level of what might be best conceptualised as 'neurocognitive resilience', and is inconsistent with a diabetic vasocognopathy model that predicts a gradual but apparently relentless decline over time as duration of hypoperfusion increases.

Also at variance with that model are results from an analysis of participants in the Leiden 85-Plus Study. Following older adults with and without type 2 diabetes over a 5-year period, van den Berg and associates (present issue of Diabetologia) [39] failed to find a faster rate of cognitive deterioration in diabetic patients over time, although at baseline (age: 85 years), diabetic patients performed more slowly on several speed tests, but this was largely attributable to their history of macrovascular disease (stroke). A greater degree of learning and memory dysfunction, generally considered to be a predictor of progression to dementia [40], was also not evident within the diabetic group. Other investigators, following somewhat younger (mean age: 75 years) adults for up to 9 years, have noted a similar pattern of results: no evidence of an accelerated decline attributable specifically to diabetes [41].

\section{A need for new research paradigms}

The absence of serious and progressive global cognitive impairment in virtually all modern studies of diabetic adults, despite the presence of clinically significant vascular complications in those samples, presents an interesting dilemma to anyone struggling to identify underlying pathophysiological mechanisms. If there is no inexorable neurocognitive deterioration in the vast majority of diabetic patients, what is it that protects the brain from further vascular (and non-vascular) damage?

Answering that question will require a number of changes in the design and conduct of neurocognitive research studies. To map the trajectory of brain changes and to differentiate age-related effects from disease duration better, longitudinal studies are needed that include both 
adults and children with carefully ascertained diabetes. Given the apparently close linkages between cognition, micro- and macrovascular diabetic complications, and diabetes-related co-morbid conditions such as hypertension, it behoves researchers studying brain function to include patients with a variety of complications rather than to exclude them, as is so often the case. This, of course, requires the use of more sophisticated and comprehensive medical (and laboratory) assessments rather than a reliance on medical record information that may be out of date or incomplete. The availability of sophisticated, non-invasive techniques to estimate the magnitude of microvascular changes within the brain, such as digital retinal imaging [15], as well as the demonstrated relationship between retinal abnormalities and brain dysfunction, make it imperative that researchers measure the retinal microvasculature at the same time a neurocognitive assessment is undertaken.

Do diabetic patients manifest a classic "diabetic encephalopathy', as described by Reske-Nielson and implied by many other writers? From this review of the recent literature, there is no evidence to support that view. Neurocognitive dysfunction does exist, but for most diabetic patients, it is likely to be quite subtle and to be manifested primarily as mental slowing, similar to the reduction in mental efficiency that occurs during the normal ageing process. Retinopathy is very clearly a marker of diabetes-associated changes in brain structure and function, and while this is consistent with a vascular pathology, the underlying mechanisms remain to be determined. Vascular and neuronal protective processes must contribute to the relative preservation of neurocognitive function, but exactly what those are remains unknown, largely because of the continuing - and perhaps misplaced - focus of diabetes researchers on brain pathology, rather than on brain protection.

\section{References}

1. Auer RN, Wieloch T, Olsson Y, Siesjo BK (1984) The distribution of hypoglycemic brain damage. Acta Neuropathol [Berl] 64:177-191

2. Auer RN, Hugh J, Cosgrove E, Curry B (1989) Neuropathologic findings in three cases of profound hypoglycemia. Clin Neuropathol 8:63-68

3. Chalmers J, Risk MTA, Kean DM, Grant R, Ashworth B, Campbell IW (1991) Severe amnesia after hypoglycemia. Diabetes Care 14:922-925

4. Gold AE, Deary IJ, Frier BM (1993) Recurrent severe hypoglycaemia and cognitive function in type 1 diabetes. Diabet Med 10:503-508

5. Perros P, Deary IJ, Sellar RJ, Best JJK, Frier BM (1997) Brain abnormalities demonstrated by magnetic resonance imaging in adult IDDM patients with and without a history of recurrent severe hypoglycemia. Diabetes Care 20:1013-1018
6. Wysocki T, Harris MA, Mauras N et al (2003) Absence of adverse effects of severe hypoglycemia on cognitive function in schoolaged children with diabetes over 18 months. Diabetes Care 26:1100-1105

7. Brands AMA, Biessels G-J, De Haan EHF, Kappelle LJ, Kessels RPC (2005) The effects of type 1 diabetes on cognitive performance: a meta-analysis. Diabetes Care 28:726-735

8. Musen G, Lyoo IK, Sparks CR, Weinger K et al (2006) Effects of type 1 diabetes on gray matter density as measured by voxelbased morphometry. Diabetes 55:326-333

9. Auer RN (2004) Hypoglycemic brain damage. Metab Brain Dis 19:169-175

10. Ryan CM, Geckle MO, Orchard TJ (2003) Cognitive efficiency declines over time in adults with type 1 diabetes: effects of microand macrovascular complications. Diabetologia 46:940-948

11. Ferguson SC, Blane A, Perros P et al (2003) Cognitive ability and brain structure in type 1 diabetes: relation to microangiopathy and preceding severe hypoglycemia. Diabetes 52:149-156

12. Wong TY, Klein R, Sharrett AR, et al (2002) Retinal microvascular abnormalities and cognitive impairment in middle-aged persons: the Atherosclerosis Risk in Communities Study. Stroke 33:1487-1492

13. Wong TY, Mosley TH, Klein R, et al, for the Atherosclerosis Risk in Communities (ARIC) Study Investigators (2003) Retinal microvascular changes and MRI signs of cerebral atrophy in healthy, middle-aged people. Neurology 61:806-811

14. Cooper LS, Wong TY, Klein R et al (2003) Retinal microvascular abnormalities and MRI-defined subclinical cerebral infarction: the Atherosclerosis Risk in Communities Study. Stroke 37:82-86

15. Patton N, Aslam T, MacGillivray T, Pattie A, Deary IJ, Dhillon B (2005) Retinal vascular image analysis as a potential screening tool for cerebrovascular disease: a rationale based on homology between cerebral and retinal microvasculatures. J Anat 206:319-348

16. Wong TY, Klein R, Klein BEK, Tielsch JM, Hubbard L, Nieto FJ (2001) Retinal microvascular abnormalities and their relationship with hypertension, cardiovascular disease, and mortality. Surv Ophthalmol 46:59-80

17. Ikram MK, De Jong FH, Van Dijk EJ et al (2006) Retinal vessel diameters and cerebral small vessel disease: The Rotterdam Scan Study. Brain 129:182-188

18. Wessels AM, Simsek S, Remijnse PL et al (2006) Voxel-based morphometry demonstrates reduced grey matter density on brain MRI in patients with diabetic retinopathy. DOI 10.1007/s00125006-0283-7

19. Suter O-C, Sunthorn T, Kraftsik R et al (2002) Cerebral hypoperfusion generates cortical watershed microinfarcts in Alzheimer disease. Stroke 33:1986-1992

20. Wessels AM, Rombouts SARB, Simsek S et al (2006) Microvascular disease in type 1 diabetes alters brain activation: a functional magnetic resonance imaging study. Diabetes 55:334-340

21. Jennings JR, Muldoon MF, Ryan C et al (2005) Reduced cerebral blood flow response and compensation among patients with untreated hypertension. Neurology 64:1358-1365

22. Schmidt R, Launer LJ, Nilsson L-G et al (2004) Magnetic resonance imaging of the brain in diabetes: The Cardiovascular Determinants of Dementia (CASCADE) Study. Diabetes 53:687-692

23. Hassing LB, Hofer SM, Nilsson SE et al (2004) Comorbid type 2 diabetes mellitus and hypertension exacerbates cognitive decline: evidence from a longitudinal study. Age Ageing 33:355-361

24. Elias PK, Elias MF, D'Agostino RB, Cupples LA et al (1997) NIDDM and blood pressure as risk factors for poor cognitive performance. Diabetes Care 20:1388-1395

25. Quirce R, Carril JM, Jiménez-Bonilla JF et al (1997) Semiquantitative assessment of cerebral blood flow with $99 \mathrm{mTc}-$ 
HMPAO SPET in type 1 diabetic patients with no clinical history of cerebrovascular disease. Eur J Nucl Med 24: $1507-1513$

26. Salem MAK, Matta LF, Tantawy AAG, Hussein M, Gad GI (2002) Single photon emission tomography (SPECT) study of regional cerebral blood flow in normoalbuminuric children and adolescents with type 1 diabetes. Pediatr Diabetes 3:155-162

27. Fülesdi B, Limburg M, Bereczki D et al (1997) Impairment of cerebrovascular reactivity in long-term type 1 diabetes. Diabetes 46:1840-1845

28. Kario K, Ishikawa $\mathrm{J}$, Hoshide $\mathrm{S}$ et al (2005) Diabetic brain damage in hypertension: role of renin-angiotensin system. Hypertension 45:887-893

29. Mäkimattila S, Malmberg-Cèder K, Häkkinen A-M et al (2004) Brain metabolic alterations in patients with type 1 diabeteshyperglycemia-induced injury. J Cereb Blood Flow Metab 24:1393-1399

30. Ruitenberg A, Den Heijer T, Bakker SLM et al (2005) Cerebral hypoperfusion and clinical onset of dementia: The Rotterdam Study. Ann Neurol 57:789-794

31. De La Torre JC (2004) Alzheimer's disease is a vasocognopathy: a new term to describe its nature. Neurol Res 26:517-524

32. De La Torre JC (2004) Is Alzheimer's disease a neurodegenerative or a vascular disorder? Data, dogma, and dialectics. Lancet Neurol 3:184-190

33. Cukierman T, Gerstein HC, Williamson JD (2005) Cognitive decline and dementia in diabetes-systematic overview of prospective observational studies. Diabetologia 48: 2460-2469

34. Biessels G-J, Staekenborg S, Brunner E, Scheltens P (2006) Risk of dementia in diabetes mellitus: a systematic review. Lancet Neurol 5:64-74

35. Reske-Nielsen E, Lundbaek K, Rafaelsen OJ (1965) Pathological changes in the central and peripheral nervous system of young long-term diabetics. Diabetologia 1:232-241

36. Meyer JS, Rauch G, Rauch RA, Haque A (2000) Risk factors for cerebral hypoperfusion, mild cognitive impairment, and dementia. Neurobiol Aging 21:161-169

37. Qiu C, Winblad B, Fratiglioni L (2005) The age-dependent relation of blood pressure to cognitive function and dementia. Lancet Neurol 4:487-499

38. Brands AMA, Kessels RPC, Biessels GJ et al (2006) Cognitive performance, psychological well-being, and brain magnetic resonance imaging in older patients with type 1 diabetes. Diabetes 55:1800-1806

39. van den Berg E, De Craen AJM, Biessels GJ, Gusselkloo J, Westendorp RGJ (2006) The impact of diabetes mellitus on cognitive decline in the oldest of the old: a prospective population-based study. Diabetologia 49:2015-2023

40. Peterson RC, Smith GE, Waring SC, Ivnik RJ, Tangalos EG, Kokmen E (1999) Mild cognitive impairment: clinical characterization and outcome. Arch Neurol 56:303-308

41. Arvanitakis Z, Wilson RS, Bienias JL, Evans DA, Bennett DA (2004) Diabetes mellitus and risk of Alzheimer disease and decline in cognitive function. Arch Neurol 61:661-666 